\title{
Assessment of occasional diathermy burn of central vessel triggered by hepatic tumor radiofrequency.
}

\author{
Zhong Jia*, Shi-Jie Li \\ Department of Hepatobiliary Surgery, Affiliated Hangzhou First People's Hospital, Zhejiang University School of \\ Medicine, Hangzhou, China
}

Keywords: Assessment, Iatrogenic injury, Therapy, Timing for surgery.

Accepted on October 30, 2018

\section{Letter to the Editor}

Radiofrequency (RF) has been confirmed to be one of the most effective treatments for malignant tumor of the liver. But occasional diathermy burn to adjacent vessels is not provisional, which sometimes may lead to undesired severe bleeding under liver capsule, even lethal consequence if conservative management is invalid for ongoing bleeding control. Hence, bleeding from capsule is becoming an occult killer [1]. Scientific assessment of diathermy burn to vessels becomes core key to save patients' lives. Obviously, the optimized timing for surgery may be prioritized concern.

To the best of our knowledge, there are three major aspects should be focused on. First and the foremost, the temperature of RF, the time of RF procedure and the length between vessels and RF zone may be the most deciding roles to influence the rupture time of involving vessel. Also, the other risk factors, including mytilus diabetes, cirrhosis and hypertension, etc., may increase fragility of vessels or opportunity of massive hemorrhage. However, interventional specialists are difficult to predict the outcome of diathermy burn to vessels ahead only depending on the potential risk factors mentioned above. Close supervision is essential measure for earlier diagnosis of diathermy burn to hepatic vessels. In practice, the first $8 \mathrm{~h}$ after RF, Post-RF d 1, and post-RF d 3 are all needed to make repeated identification for RF zone and adjacent vessels. No previous studies revealed which length between tumor margin and its adjacent vessels is safe. In authors' opinion, the length between vessels to the RF needle top should not be less than $5.0 \mathrm{~cm}$.

Secondly, prevention of diathermy burn is easier than treatment of that because diathermy burn is a complex injury. Hence, it's not easy to self-recovery. As a result, delayed rupture of vessel due to sequela following burn injury may bring about deadly hit for patients underwent RF. Regarding this point, it's necessary to make an earlier accurate assessment in some key points, particularly for high-risk patients. "Wait-to-see" or excessive earlier or progressive surgical intervention may both be improper due to their potential risks of life threatening. For high-risk patients, 3D reconstruction of liver vessels based on computed tomography data ought to be conducted if necessary.
Once post-RF diathermy burn of vessel is suspected or has already been confirmed, please do not hesitate to perform reevaluation of imaging so as to earlier addressing the injured location and its severity extent. And judge the type of liver trauma and its severity degree according to liver trauma classification [2].

Thirdly, how to assess the extent of severity of bleeding based on clinical presentations? These signals, including upper quadrant abdominal dull pain or increasing distention, declined hemoglobin, instability of hemodynamic, etc., are all a meaningful alerts. Generally, it's a little too late to save one's life once shock suddenly occurs.

What time for surgery remains an issue due to controversial exiting in various expertises. In general, Child-Pugh score $(>7)$ or model of end liver disease (MELD) score $(>11)$ for patients with liver trauma or iatrogenic injuries are climbed, the therapy with transcatheter arterial embolization (TAE) may be safer compared to surgery [3]. But if you have no way to stop bleeding, mesh-wrapping [4] or gauze-packing during surgery is a possible remedy to stop intrahepatic ongoing bleeding.

Back on authors' experiences, raising awareness of adverse effect in RF procedure is more important than focusing on assessment or therapy alone.

\section{References}

1. Luo MY, Jia Z, Cai Y, Wan YF. Bleeding from the liver capsule-when to perform surgery. Chin Med J 2017; 130: 1008.

2. Coccolini F, Catena F, Moore EE. WSES classification and guidelines for liver trauma. World J Emerg Surg 2016; 11: 50 .

3. Yu WY, Li QJ, Gong JP. Treatment strategy for hepatic trauma. Chinese J Traumatol 2016; 19: 168-171.

4. Dellaportas D, Nastos C, Psychogiou V. Iatrogenic liver trauma managed with mesh-wrapping and ligation of portal vein branch: a case report. Int J Surg Case Rep 2011; 2: 261-263. 


\section{*Correspondence to}

Zhong Jia

Department of Hepatobiliary Surgery

Affiliated Hangzhou First People's Hospital

Zhejiang University School of Medicine

PR China 